\title{
A bioinformatics approach to distinguish plant parasite and host transcriptomes in interface tissue by classifying RNA-Seq reads
}

Daisuke Ikeue ${ }^{1}$, Christian Schudoma ${ }^{2,5}$, Wenna Zhang ${ }^{2}$, Yoshiyuki Ogata ${ }^{1}$, Tomoaki Sakamoto ${ }^{3}$, Tetsuya Kurata ${ }^{3}$, Takeshi Furuhashi ${ }^{4,6}$, Friedrich Kragler ${ }^{2}$ and Koh Aoki ${ }^{{ }^{*}}$

\begin{abstract}
Background: The genus Cuscuta is a group of parasitic plants that are distributed world-wide. The process of parasitization starts with a Cuscuta plant coiling around the host stem. The parasite's haustorial organs then establish a vascular connection allowing for access to the phloem content. The host and the parasite form new cellular connections, suggesting coordination of developmental and biochemical processes. Simultaneous monitoring of gene expression in the parasite's and host's tissues may shed light on the complex events occurring between the parasitic and host cells and may help to overcome experimental limitations (i.e. how to separate host tissue from Cuscuta tissue at the haustorial connection). A novel approach is to use bioinformatic analysis to classify sequencing reads as either belonging to the host or to the parasite and to characterize the expression patterns. Owing to the lack of a comprehensive genomic dataset from Cuscuta spp., such a classification has not been performed previously.

Results: We first classified RNA-Seq reads from an interface region between the non-model parasitic plant Cuscuta japonica and the non-model host plant Impatiens balsamina. Without established reference sequences, we classified reads as originating from either of the plants by stepwise similarity search against de novo assembled transcript sets of $C$. japonica and I. balsamina, unigene sets of the same genus, and CDNA sequences of the same family. We then assembled de novo transcriptomes from the classified read sets. We assessed the quality of the classification by mapping reads to contigs of both plants, achieving a misclassification rate low enough (0.22-0.39\%) to be used reliably for differential gene expression analysis. Finally, we applied our read classification method to RNA-Seq data from the interface between the non-model parasitic plant $C$. japonica and the model host plant Glycine max. Analysis of gene expression profiles at 5 parasitizing stages revealed differentially expressed genes from both C. japonica and G. max, and uncovered the coordination of cellular processes between the two plants.

Conclusions: We demonstrated that reliable identification of differentially expressed transcripts in undissected interface region of the parasite-host association is feasible and informative with respect to differential-expression patterns.
\end{abstract}

Keywords: Cuscuta japonica, Cuscuta reflexa, Classification, Parasitic plant, Transcriptome, Parasite-host interaction

\footnotetext{
*Correspondence: kaoki@plant.osakafu-u.ac.jp

${ }^{1}$ Graduate School of Life and Environmental Sciences, Osaka Prefecture

University, 1-1 Gakuen-Cho, Naka-Ku, Sakai, Osaka 599-8531, Japan

Full list of author information is available at the end of the article
} 


\section{Background}

In angiosperms, approximately 4000 species are parasitic to some extent [1]. Parasitic plants have evolved from at least 11 independent clades [2]. They depend, partly or entirely, on a host plant for acquisition of water and nutrients. The ability to consume water and nutrients from the host plant affects the appearance and metabolism of parasitic plants. In general, parasitic plants either partially lost the capacity of photosynthetic production (hemiparasitic plants) or entirely depend on host plants (holoparasitic plants) [3].

The genus Cuscuta is a prominent group of parasitic plants. It consists of 150-200 species that are distributed world-wide [4]. Some Cuscuta spp. are known to infest fields, thereby leading to crop losses. Although seedlings of Cuscuta are self-sufficient, mature plants have no roots, and their leaves are reduced to small scales. Parasitism of Cuscuta starts with sensing the host plant and coiling around the host stem. This action is followed by formation of prehaustorium structures from meristematic cells [5]. Invasion of the host tissue by the haustorium is initiated by production of a set of enzymes degrading the host cell wall [6] and inducing a host defense response (also reported for herbivores and pathogens [7]). According to the degree of defense response of the host plant to prevent the haustorium from reaching the vascular tissue or from establishing a functional conduit, the interaction between parasite and host plants can be classified as compatible or incompatible [8,9]. In a compatible host, a Cuscuta-host feeding connection is usually established by the formation of new vascular tissue connecting the pre-existing host vasculature to the Cuscuta vasculature. Dye tracer experiments showed both an apoplastic [8] and a symplastic exchange [10] of small molecules between the species. Additionally, the transfer of macromolecules such as mRNA [11,12], and siRNA [13] as well as viruses [14] indicates the existence of a symplastic parasite-host interface. Furthermore, microscopy studies demonstrated the presence of protoxylem cells in the interface between Cuscuta and host tissues [8]. Even when Cuscuta attaches itself to an incompatible host, transfer cells specializing in water and nutrient uptake are initiated at the interface, but the transfer of nutrients via the phloem sieve tube does not occur [8]. Obviously, in both compatible and incompatible interactions, tight coordination of growth and differentiation between a parasite plant and its host is essential. It is challenging, however, to assign the underlying molecular events to specific cells belonging to Cuscuta or its host.

The formation of these cellular structures at the cell-tocell interface seems to tighten the physical connection, thus making it difficult to detach cells of the parasite from host cells to investigate gene expression profiles of respective plants. Given that morphological markers exist, the individual tissues can be isolated using laser microdissection and subjected to RNA-Seq (whole-transcriptome shotgun sequencing) analysis [15]. Nevertheless, the Cuscuta tissue at the interface represents a highly complex branched structure composed of haustorial tissue and searching hyphae [16]. Thus, in most instances, this tissue is too complex to be dissected and analyzed in a simple fashion. An alternative method could be to classify RNA sequencing data using a bioinformatics approach. For instance, in transcriptomic analysis of Cuscuta pentagona using RNA-Seq (whole-transcriptome shotgun sequencing), reads originating from the host plant were removed using the reference sequences of compatible hosts $[15,17,18]$. In the analysis of RNA movement between C. pentagona and host plants (Arabidopsis and tomato), similar read classification based on the similarity to the host's reference sequences was performed to distinguish transcripts from parasite plant and host plant [12]. Since complete genome sequences for Cuscuta spp. and their natural hosts are not available, the above classification and filtering cannot be used. However, the latest nextgeneration sequencing technology provides sufficient depth (numbers of reads) and sequence length to classify reads and to identify specific expression patterns.

In this study, we describe a bioinformatics approach to classify RNA-Seq reads obtained from an interface region formed between the non-model parasite plant Cuscuta japonica and the non-model host plant Impatiens balsamina. Without established reference sequences, we classified RNA-Seq reads using a stepwise classification procedure by means of similarity search to i) sequences obtained from tissues harvested from non-feeding $C$. japonica and I. balsamina, ii) sequences of plants belonging to the same genus, particularly of $C$. reflexa which is the phylogenetically closest species to C. japonica [4] and iii) sequences of the same family. The filtered sequences were used for de novo transcriptome assembly of tissues consisting of cells from C. japonica and I. balsamina. Using a competitive mapping approach in which reads were mapped to contigs of both plants, we quantitatively assessed the probability of misclassification. We achieved a misclassification rate low enough (0.22-0.39\%) to avoid a significant difference of accuracy in identifying differentially expressed genes compared to conventional mapping. We then applied the read classification strategy to the RNA-Seq data from the interface between C. japonica and a model plant, Glycine max (soybean). RNA-Seq reads obtained from $C$. japonica-G. max interface regions at 5 parasitizing stages were subjected to the read classification, and genes regulated in a stage-dependent manner were identified both for C. japonica and G. max. The read classification method presented here will be useful for analyzing other multiorganism systems. 


\section{Results and discussion Classifying RNA-Seq reads derived from two non-model plants}

We attempted to identify genes expressed in the interface region formed between two non-model plants, the parasite C. japonica, and the host, I. balsamina (Figure 1). Our first aim was to assemble de novo transcriptome sets using RNA-Seq reads obtained from the interface region without physically dissecting either the parasite's or host's tissues. Instead of using physical dissection, we employed a bioinformatic approach to classify the reads into two groups; 1) C. japonica, and 2) I. balsamina. As references for classification of the reads, sequence sets of the transcriptome were assembled from samples of $C$. japonica and $I$. balsamina that grew independently and were not in contact (nc; Figure 2). Sets of RNA-Seq reads derived from C. japonica (Cj_nc_reads) and I. balsamina (Ib_nc_reads)

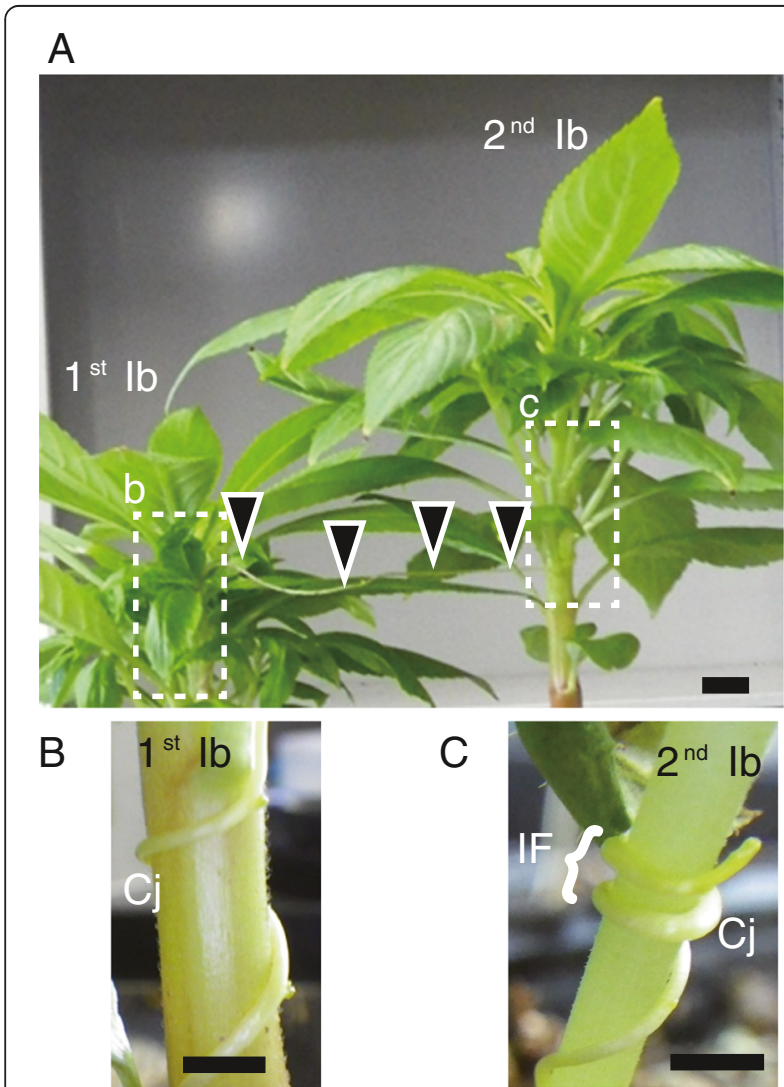

Figure 1 Experimental setup of C. japonica-I. balsamina association. (A) C. japonica (Cj) made parasite-host association to the first I. balsamina individual ( $1^{\text {st }} \mathrm{lb}$, left). Elongated $C$. japonica attached to the second I. balsamina individual $\left(2^{\text {nd }} \mathrm{lb}\right.$, right) and made parasite-host association. Interface region (IF) was sampled from the second I. balsamina ( $2^{\text {nd }} \mathrm{lb}$, right). Black triangles indicate an elongated stem of C. japonica. (B) Magnified image of the interface region on the stem surface of the first $I$. balsamina $\left(1^{\text {st }} \mathrm{lb}\right)$. (C) Magnified image of the interface region of the second I. balsamina $\left(2^{\text {nd }} \mathrm{lb}\right)$. Tissue indicated with a white curly bracket (IF) was sampled as the interface region tissue. Scale bars indicate $1 \mathrm{~cm}$. can be considered non-contaminated. We assembled the reads from these sets into $62,648 \mathrm{Cj}$ _nc_contigs and 25,301 Ib_nc_contigs, respectively. The difference in contig numbers could be due to the tissue specificity of the gene expression in the C. japonica subapical stem and the I. balsamina stem.

The reads obtained from the interface region (Figure $1 C$ ) in which C. japonica parasitized to I. balsamina (CjIb_if_reads; Figure 2) were classified using three sequential rounds of mapping. First, we mapped the CjIb_if_reads separately to the contigs from tissues that were not-in-contact, $\mathrm{Cj}$ _nc_contigs and Ib_nc_contigs. Reads that were uniquely mapped onto either $\mathrm{Cj}_{-} \mathrm{nc}$ contigs or Ib_nc_contigs were classified as originating from C. japonica or I. balsamina, respectively (Figure 2). The reads that remained unmapped at this point likely contained transcripts specific to the interface region. To distinguish $C$. japonica reads from I. balsamina reads, we used previously published unigene sets of $C$. pentagona and $C$. suaveolens $[17,18]$. In addition, we constructed a novel contig set of $C$. reflexa, the species phylogenetically closest to C. japonica [4]. This analysis was performed using RNA-Seq reads from self-parasitizing tissue in which the subapical region of $C$. reflexa formed haustorial connections to distant parts of its own stem or to other C. reflexa individuals feeding on tomato. The C. reflexa transcriptome was assembled from 308,147,540 pairedend reads and consisted of 165,213 contigs (Cr_contigs, Table 1). We performed the second classification by mapping the reads that remained unmapped in the first classification onto these Cuscuta-genus unigene sets. The mapping reads were then considered as originating from C. japonica (11,001,253 reads). During the final step, we mapped the remaining reads to the NCBI nt (nucleotide) database. If reads mapped to nucleotide entries from Convolvulaceae or Balsaminaceae families, they were considered to be derived from C. japonica or I. balsamina, respectively. This step revealed additional 26,045 C. japonica and 9,393 I. balsamina reads.

After this stepwise classification, $73 \% \quad(75,842,928$ reads) of the sequence information from the interface region $(104,521,554$ CjIb_if_reads) could be mapped to C. japonica, whereas $8.2 \%(8,502,752$ reads $)$ mapped to I. balsamina (Figure 2). This difference in the numbers of classified reads was probably due to difference in the concentration of RNA in the parasite and the host tissues. The amount of total RNA per mg fresh weight of not-in-contact stem tissue of C. japonica was $16.8 \pm 6.5$ fold greater than that in an I. balsamina sample of equal mass (Additional file 1). Thus, in the interface-region samples that contained nearly equal amounts of parasite and host tissues, a larger number of $C$. japonica reads was expected. In a previous study on the associations between C. pentagona and Arabidopsis and C. pentagona 


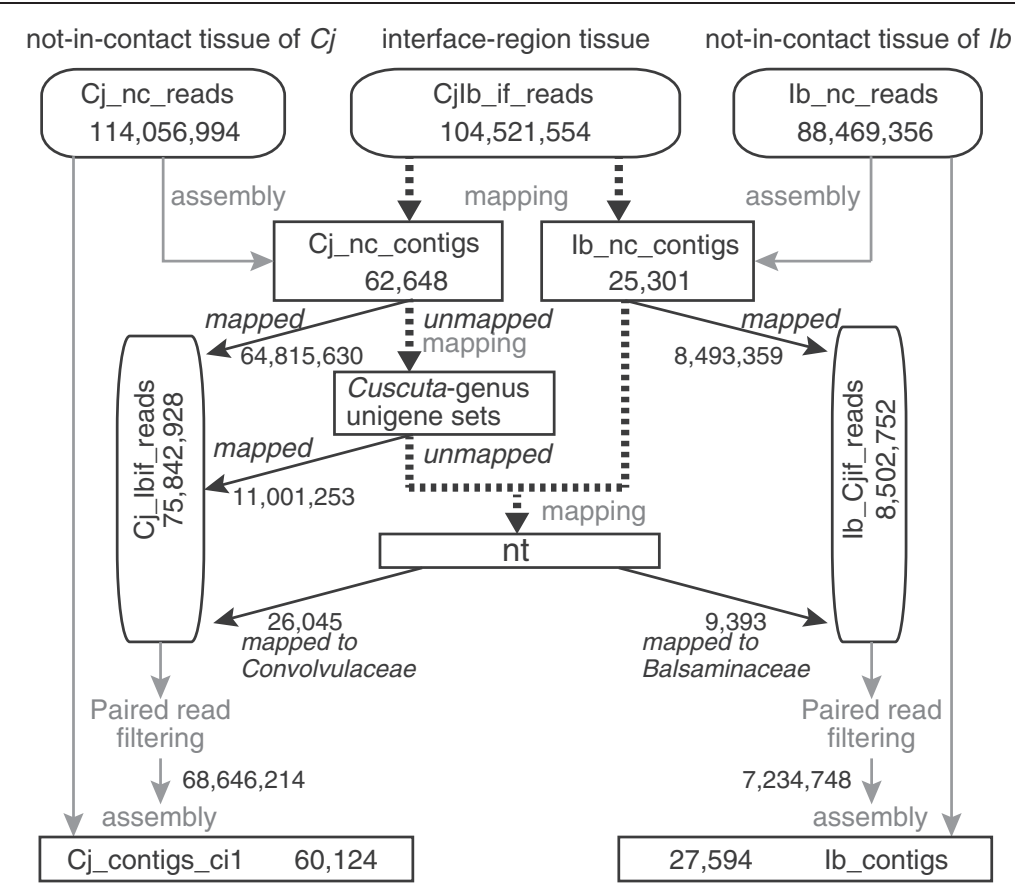

Figure 2 Schematic diagram of classification of reads. Dotted lines indicate that mapping was performed. Solid lines indicate that assembly or classification was performed. Abbreviations: Cj, C. japonica; lb, I. balsamina; nc, not-in-contact; if, interface region. Numbers indicate the numbers of reads.

and tomato, the interface regions contained higher portions of host reads (51\% from Arabidopsis and 86\% from tomato) [12]. The discrepancy with the present study might be due to the fact that the interface region in this study was sampled from the stem of $C$. japonica closer to the apex (1 cm from the tip) than the region used in the C. pentagona study ( $>7.5 \mathrm{~cm}$ from the tip) [12], and therefore resulted in a higher percentage of $C$. japonica reads.

\section{De novo assembly and annotation}

The classified reads from the interface region $\left(\mathrm{Cj}_{\text {_Ibif_- }}\right.$ reads and Ib_Cjif_reads) were merged with the respective read set from not-in-contact tissues $\left(\mathrm{Cj}_{-}\right.$nc_reads or Ib_nc_reads). The merged read sets were used for de novo transcriptome assembly using Velvet/Oases $[19,20]$.
The assembled C. japonica and I. balsamina transcriptomes consisted of 60,124 contigs (Cj_contigs_ci1, median length $599 \mathrm{bp}$, average length $980 \mathrm{bp}$; Table 1) and 27,594 contigs (Ib_contigs, median length $1,026 \mathrm{bp}$, average length 1,276 bp; Table 1).

The C. reflexa transcriptome used in read classification was based on 308,147,540 paired-end reads as input for de novo assembly using Trinity (version r20140717) [21]. The raw assembly consisted of 165,213 Cr_contigs $(246,886$ transcript variations, with median length $377 \mathrm{bp}$, average length 620 bp; Table 1). We could predict ORFs in 89,456 C. reflexa contigs (median length $330 \mathrm{bp}$ ). Of these contigs, 64,442 contained full-length ORFs (median length $363 \mathrm{bp}$ ).

These numbers of assembled transcripts may be an overestimation for the actual numbers of genes expressed

Table 1 De novo assembly and annotation of $C$. reflexa, $C$. japonica, and $I$. balsamina contigs

\begin{tabular}{llll}
\hline & C. reflexa (Cr_contigs) & C. japonica (Cj_contigs_ci1) & I. balsamina (Ib_contigs) \\
\hline Library type & Illumina, $90 \mathrm{bp}$ paired-end & Illumina, 101 bp paired-end & Illumina, 101 bp paired-end \\
Assembler & Trinity & Velvet/Oases & Velvet/Oases \\
Assembled reads & $308,147,540$ & $182,703,208$ & $95,704,104$ \\
Number of contigs & 165,213 & 60,124 & 27,594 \\
Median contig length & $377 \mathrm{bp}$ & $599 \mathrm{bp}$ & $1,026 \mathrm{bp}$ \\
Average contig length & $620 \mathrm{bp}$ & $980 \mathrm{bp}$ & $1,276 \mathrm{bp}$ \\
ORF predicted & $89,456(54 \%)$ & $59,768(99 \%)$ & $27,507(99 \%)$ \\
Full-length ORF & $64,442(39 \%)$ & $33,313(55 \%)$ & $18,118(66 \%)$ \\
\hline
\end{tabular}


in the tissues. This overestimation is possibly due to the presence of alleles, alternatively spliced transcripts, and fragmented transcripts. Further refinement will be required on the basis of annotation.

The gene ontology (GO) category distributions for Cj_contigs_ci1 and Ib_contigs, based on the similarity to the RefSeq database [22] and TAIR10 [23], did not have a bias toward any specific categories (Additional file 2). A similarity search for transcripts of other plants revealed that $C$. japonica transcripts showed the highest similarity to C. reflexa, and showed lower similarity to parasitic plants belonging to the Orobanchaceae family (Additional file 3).

\section{Quality assessment of read classification}

The transcripts of $C$. japonica and I. balsamina were de novo assembled from all reads including those obtained from the interface region. This fact prompted us to estimate the extent of misclassification of $C$. japonica reads as I. balsamina transcripts or vice versa. To this end, we performed a competitive mapping. In conventional cases, RNA-Seq reads obtained from an organism are mapped solely onto the reference sequence of that organism. Here, our strategy was to map reads onto both C. japonica and I. balsamina contigs. A binary choice was made based on a higher mapping score (identity and $e$ value) in order to assign reads to one of the two species. Since using reads from the interface-region samples would make it harder to properly discriminate the true and false source organisms, we used only $\mathrm{Cj}_{-}$nc_reads and Ib_nc_reads for our estimation.

Misclassification during mapping will inevitably occur due to the presence of homologous transcripts between C. japonica and I. balsamina (Additional file 3). To estimate the extent to which this misclassification can be attributed to the presence of homologous transcripts, we mapped $\mathrm{C}$ _nnc_reads and Ib_nc_reads to a merged dataset consisting of $\mathrm{Cj} \_n c \_c o n t i g s$ and Ib_nc_contigs (Figure 3A). Because Cj_nc_contigs and Ib_nc_contigs were assembled separately from the two nonoverlapping read sets, any instance of misclassification had to occur due to the presence of identical sequences in the parasite and the host. According to this test, the background rates of false classification were revealed as: $2.38 \%$ (Cj_nc_reads mapped to Ib_nc_contigs) and $0.22 \%$ (Ib_nc_reads mapped to Cj_nc_contigs; Table 2A).

In comparison with this mapping, we mapped Cj_nc_reads and Ib_nc_reads to a merged transcript set

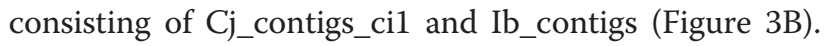
During this round of mapping, an instance of false assignment could be attributed to read misclassification that occurred prior to the assembly of $\mathrm{Cj}_{\text {_contigs_ci1 }}$ and Ib_contigs, in addition to transcript homology. Here, $0.39 \%$ of $\mathrm{C}$ _nc_reads were mapped to Ib_contigs and
$0.31 \%$ of $\mathrm{Ib}$ nc_reads were mapped to $\mathrm{C}$ _contigs_ci1 (Table 2B), suggesting that these cross-mapped reads were misclassified. We estimated the rate of false classification under more stringent conditions where only exact matches were allowed. The frequency of false assignments of Cj_nc_reads to Ib_contigs decreased to $0.28 \%$ and that of Ib_nc_reads to Cj_contigs_cil decreased to $0.22 \%$ (Table $2 \mathrm{C}$ ).

Finally, we evaluated the quality of the classification by analyzing the receiver operating characteristic (ROC) and its area under the curve (AUC) [24]. The AUC approaches 1.0 when better classification is achieved with a greater ratio of true positive to false positive results. When nc_reads were mapped onto nc_contigs, the AUC was 0.981 (Table 2A). When nc_reads were mapped onto contigs assembled from classified reads, we obtained a higher AUC of 0.995 (Table 2B).

These results collectively demonstrated that, when we mapped the reads obtained from the interface region to the transcripts assembled from them, these reads could be assigned to the wrong plant. Nevertheless, according to the ROC AUC, read classification performed prior to transcript assembly did not impair the binary choice more strongly than in the case of false assignments solely due to the presence of homologous transcripts. These results raise the question whether false assignments can happen more frequently between plant species that are more closely related. A systematic analysis of RNA-Seq reads from many plant species will be necessary to find an answer to this question.

We next tested whether or not including misclassified

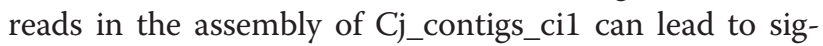
nificantly different results with respect to the identification of differentially expressed genes between not-incontact and interface-region tissues. To test this idea, we analyzed two different mapping results to identify differentially expressed genes (Additional file 4). First, Cj_nc_reads and Cj_Ibif_reads were separately mapped to Cj_contigs_ci1. Differentially expressed genes were then identified by comparison of the two mapping results. The same procedure was applied to Ib_nc_reads and Ib_Cjif_reads by separately mapping them onto Ib_contigs (Figure 3C). Second, to exclude potentially misclassified reads from the estimation of gene expression, $\mathrm{Cj}_{\text {_nc_reads }}$ and $\mathrm{Cj}$ _Ibif_reads were separately mapped to a transcript set containing both $\mathrm{Cj}_{-}$contigs_cil and Ib_contigs (Figure 3D). Ib_nc_reads and Ib_Cjif_reads were also separately mapped to the same merged contig set (Figure 3D). Here, reads that were mapped to the wrong set (C. japonica reads to I. balsamina contigs or vice versa) were excluded from the RPKM-estimation. Both approaches yielded identical estimates for the number of differentially regulated transcripts in the interface region, except for the number of 


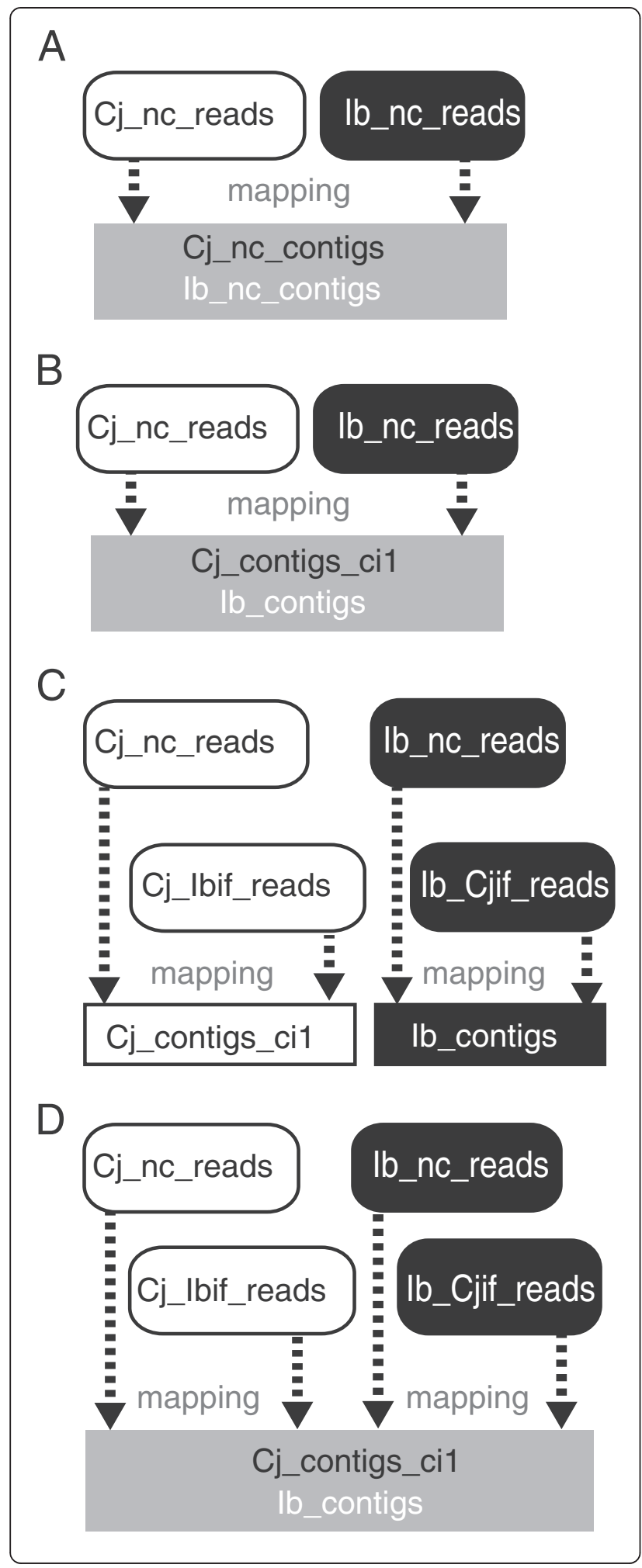

Figure 3 Scheme of read mapping to estimate the rate of false classification. (A) Cj_nc_reads and Ib_nc_reads were mapped to a merged transcript set of Cj_nc_contigs and Ib_nc_contigs. (B) Cj_nc_reads and Ib_nc_reads were mapped to a merged transcript set of Cj_contigs_cil and Ib_contigs. (C) Cj_nc_reads and Cj_lbif_reads were mapped onto Cj_contigs_ci1. Ib_nc_reads and Ib_Cjif_reads were mapped onto Ib_contigs. (D) Cj_nc_reads, Cj_Ibif_reads, Ib_nc_reads and Ib_Cjif_reads were mapped onto a merged transcript set of Cj_contigs_cil and Ib_contigs. The result of the mapping in panel (C) was used for the identification of differentially expressed genes.

down-regulated genes in C. japonica (Table 3). This result implies that excluding potentially misclassified reads makes no significant difference for the identification of transcripts differentially expressed in the interface region.

\section{Application of the read classification to C. japonica-model plant interaction}

We applied the read classification method to RNA-Seq data obtained from the interface region between $C$. japonica and a model-plant host, Glycine max (soybean) whose reference transcriptome was available [25] (Figure 4). Interface regions were sampled from five parasitizing stages at $24 \mathrm{~h}$ after attachment (haa), 48 haa, 72 haa, 96 haa and 120 haa (Figure 4A). Reads from interface regions were classified using two approaches. The "stepwise classification" approach (Figure $5 \mathrm{~A}$ ) was based on the reference transcriptome of G. max, Gmax_275_Wm82.a2.v1.transcript [26] to select G. $\max$ reads, and used the read classification method presented in the previous sections to identify C. japonica reads. By contrast, the "reference-based-classification" approach (Figure 5B), mapped reads from the interface regions onto a reference transcriptome set of G. max. Reads that mapped to the reference were regarded as G. max reads, non-mapping reads were assumed to originate from C. japonica.

C. japonica reads from the five parasitizing stages were pooled and subjected to de novo transcriptome assembly. Reads obtained by stepwise classification were assembled into 59,449 contigs (Cj_contigs_cg1, Table 4). On the other hand, reads obtained by reference-based classification were assembled into 249,621 contigs (Cj_contigs_cg2, Table 4). A larger number of short contigs (i.e. <200 bp) that did not have similarity to the RefSeq entries was present in Cj_contigs_cg2 than Cj_contigs_cg1 (Table 4). This result implied that the quality of the contig set obtained by stepwise classification was better than that obtained by reference-based classification.

Next, we compared the probability of misclassification between the two approaches. We performed a competitive mapping of reads obtained from not-in-contact

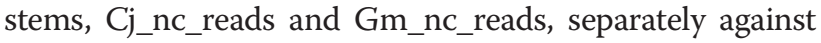
merged contig sets consisting of the G. $\max$ reference transcriptome and either Cj_contigs_cg1 or Cj_contigs_cg2. 
Table 2 Assessment for the rate of misclassification of reads by mapping Cj_nc_reads and Ib_nc_reads onto two transcript sets

\begin{tabular}{|c|c|c|c|c|}
\hline \multirow[t]{7}{*}{ A. } & Contig set & \multicolumn{3}{|c|}{ Merged set of Cj_nc_contigs and lb_nc_contigs } \\
\hline & Classification & Reads mapped onto C. japonica (\%) ${ }^{a}$ & Reads mapped onto I.balsamina (\%) ${ }^{a}$ & $A \cup C^{b}$ \\
\hline & Mapped reads & \multicolumn{2}{|l|}{ Number of reads (\%) } & \multirow[t]{5}{*}{0.981} \\
\hline & \multirow[t]{2}{*}{ Cj_nc_reads } & $76,775,986$ & $2,712,459$ & \\
\hline & & $(67.3)$ & $(2.38)$ & \\
\hline & \multirow[t]{2}{*}{ Ib_nc_reads } & 195,363 & $71,599,224$ & \\
\hline & & $(0.22)$ & $(80.9)$ & \\
\hline \multirow[t]{7}{*}{ B. } & Contig set & \multicolumn{3}{|c|}{ Merged set of Cj_contigs_cil and Ib_contigs } \\
\hline & Classification & Reads mapped onto C. japonica (\%) ${ }^{a}$ & Reads mapped onto I.balsamina (\%) ${ }^{a}$ & $A \cup C^{b}$ \\
\hline & Mapped reads & \multicolumn{2}{|l|}{ Number of reads (\%) } & \multirow[t]{5}{*}{0.995} \\
\hline & \multirow[t]{2}{*}{ Cj_nc_reads } & $73,819,654$ & 442,526 & \\
\hline & & $(64.7)$ & $(0.39)$ & \\
\hline & \multirow[t]{2}{*}{ Ib_nc_reads } & 271,929 & $71,471,262$ & \\
\hline & & $(0.31)$ & $(80.8)$ & \\
\hline \multirow[t]{7}{*}{ C. } & Contig set & \multicolumn{3}{|c|}{ Merged set of Cj_contigs_cil and Ib_contigs } \\
\hline & Classification & Reads mapped onto C. japonica (\%) ${ }^{a}$ & Reads mapped onto I.balsamina (\%) ${ }^{a}$ & $A \cup C^{b}$ \\
\hline & Mapped reads & \multicolumn{2}{|l|}{ Number of reads (\%) } & \multirow[t]{5}{*}{0.995} \\
\hline & \multirow[t]{2}{*}{ Cj_nc_reads } & $58,501,463$ & 324,744 & \\
\hline & & $(55.3)$ & $(0.28)$ & \\
\hline & \multirow[t]{2}{*}{ Ib_nc_reads } & 197,487 & $60,709,899$ & \\
\hline & & $(0.22)$ & (68.6) & \\
\hline
\end{tabular}

${ }^{a}$ Numbers indicate percentage of mapped reads to the total number of resds. ${ }^{b} \mathrm{AUC}$; area under receiver operating characteristic (ROC) curve. Mapping parameter was as follows; In Panel A and Panel B, match length $\geq 90$ bp and at most 1 mismatch and 1 gap allowed. In Panel C, match length $=100$ bp and no mismatch allowed.

Using $\mathrm{Cj}$ _contigs_cg1 resulted in a higher AUC value

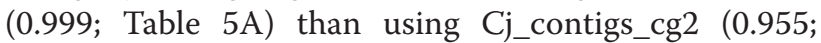
Table $5 \mathrm{~B}$ ), suggesting that the stepwise classification approach is better.

For the detection of differentially expressed genes during establishment of parasitic connection, the Cj_contigs_cg1 provided a more robust result than the $\mathrm{Cj}_{\text {_contigs_cg2 }}$ (Table 6). We compared the number of detected differentially expressed genes by either i) mapping reads classified from the interface region (Cj_Gmif_reads and Gm_Cjif_ reads) separately to the C. japonica and G. max contig

Table 3 Assessment of the accuracy in identifying differentially expressed genes in parasitic tissue

\begin{tabular}{lll}
\hline Mapping procedure & $\begin{array}{l}\text { Separately onto } \\
\text { Cj_contigs_ci1 } \\
\text { and Ib_contigs }\end{array}$ & $\begin{array}{l}\text { To a merged set } \\
\text { of } \mathbf{C j} \text { _contigs_ci1 } \\
\text { and Ib_contigs }\end{array}$ \\
Differential expression & $\begin{array}{l}\text { number of contigs } \\
\text { number of contigs }\end{array}$ \\
\hline Upregulated in C. japonica & $284^{\mathrm{a}}$ & $284^{\mathrm{a}}$ \\
Downregulated in C. japonica & $944^{\mathrm{b}}$ & $940^{\mathrm{b}}$ \\
Upregulated in I. balsamina & $10^{\mathrm{a}}$ & $10^{\mathrm{a}}$ \\
Downregulated in I. balsamina & $530^{\mathrm{a}}$ & $530^{\mathrm{a}}$ \\
\hline
\end{tabular}

${ }^{a}$ Member contigs are the same in all classes. ${ }^{b} 939$ transcripts were found in common. ${ }^{c}$ Corresponds to Figure $3 C$. ${ }^{d}$ Corresponds to Figure 3D. sets, or ii) by mapping Cj_Gmif_reads and Gm_Cjif_reads separately to a merged contig set consisting of $C$. japonica contigs and G. $\max$ contigs. When combining $\mathrm{Cj}_{\text {_con- }}$ tigs_cg1 with the G. $\max$ reference transcriptome, the differences in numbers of differentially expressed genes between the two cases $(17,653$ and 17,656; Table 6) were smaller than when combining $\mathrm{Cj}$ _contig_cg2 with the $G$. $\max$ reference transcriptome (17,653 and 17,526; Table 6). Collectively, these results confirmed that the stepwise classification approach resulted in a better de novo transcriptome assembly, at least in the case of $C$. japonica, with respect to the quality of the contig set and robustness in the identification of differentially expressed genes.

\section{Comparative gene expression profiling of the parasite plant and the host plant}

Distinguishing read origins via classification allowed us to simultaneously monitor gene expression profiles of both $C$. japonica and G. max in the interface region. We used $\mathrm{C}$ __contigs_cg1 together with the $G$. max reference transcriptome for mapping reads from the interface region, and identified differentially expressed transcripts (Additional file 5). Expression profiles of all differentially expressed transcripts, 3,819 C. japonica contigs and 17,653 


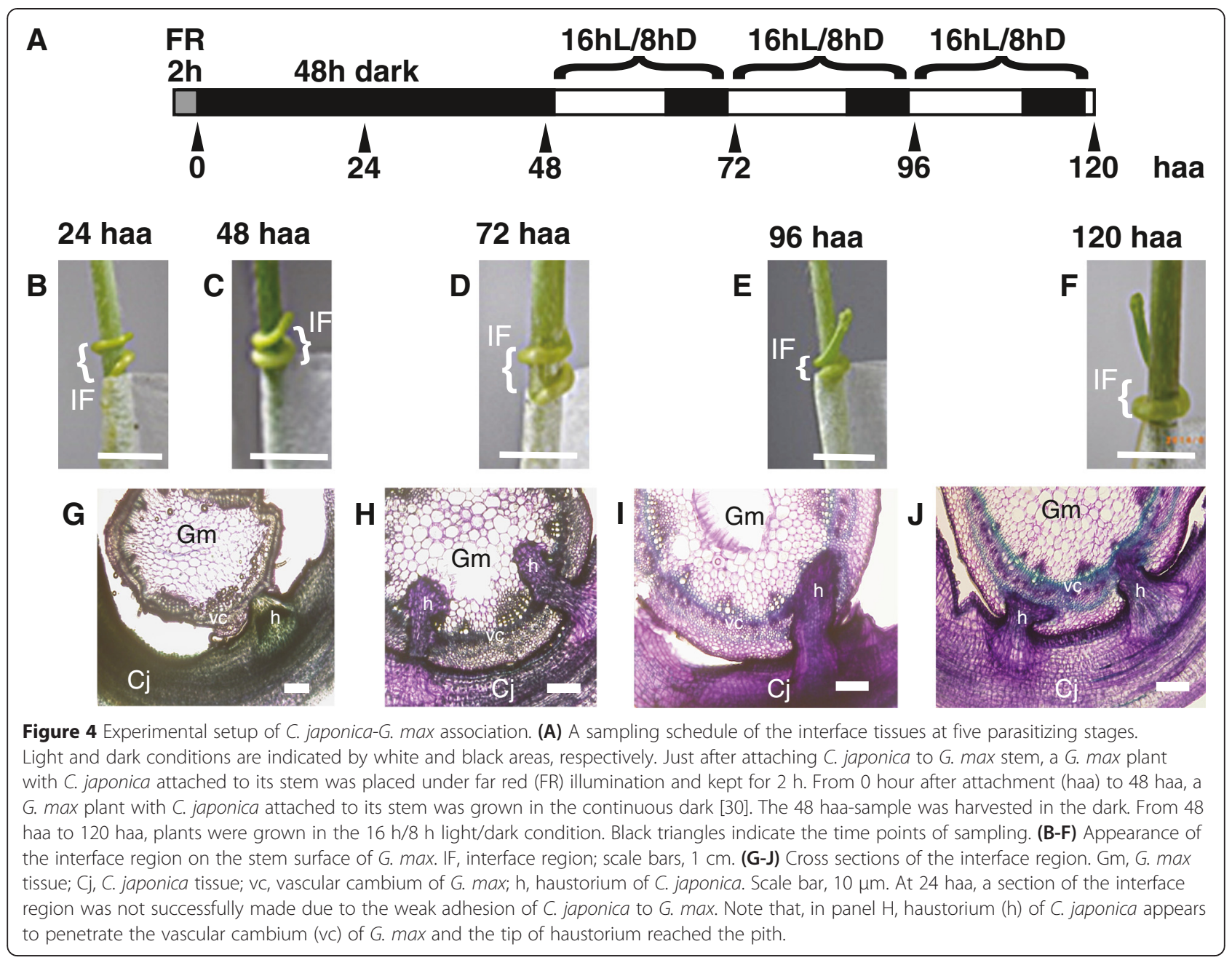

G. $\max$ contigs, were collectively subjected to cluster analysis and classified into 10 clusters according to the expression patterns across the stages (Table 7). Each parasitizing stage was characterized as follows. At 24 haa, C. japonica had completed coiling around G. $\max$ stem, and prehaustorium structures formed (Figure 4B). At 48 haa, elongation of $C$. japonica was arrested, and the tip of the haustorium was localized in the cortex (Figure $4 \mathrm{C}$ and G). At 72 haa, elongation of C.japonica was still arrested, and the tip of the haustorium was localized in the pith (Figure 4D and $\mathrm{H}$ ). At 96 haa, elongation of C.japonica restarted, and the tip of the haustorium was localized at the vascular cambium (Figure 4E and I). At 120 haa, the stem of $C$. japonica elongated, and and the tip of the haustorium still localized at the vascular cambium (Figure $4 \mathrm{~F}$ and $\mathrm{J}$ ). We focus here on the 6 clusters (cluster1, cluster 3 , cluster 7 , cluster 2 , cluster 5 and cluster 4 ) whose expression profile peaked at one of the 5 stages (Figure 6A).

In several GOslim categories enriched in these stages, we found similarities as well as differences in the composition of underlying transcripts between $C$. japonica and G. $\max$ (Figure 6B). Under the GOslim category of "Hydrolase Activity", transcripts encoding carbohydrate-, lipid- and protein-degrading enzymes were consistently found in cluster 1 and cluster 2 of $C$. japonica. On the other hand, in cluster 2 of G. max, the number of contigs related to the ubiquitin-dependent protein catabolic process increased transiently. In cluster 4 of G. $\max$, the number of contigs associated with defense response increased, i.e., disease resistance protein (TIR-NBS-LRR class) family. A fact that approximately $44 \%$ of $C$. japonica contigs and $36 \%$ of G. max contigs in this category were associated with "Extracellular Region", "Cell Wall" and "Plasma Membrane" reinforced the notion that various molecular interactions occur in the extracellular region between parasite and host. Expression of ubiquitin-proteasome pathway genes can be regarded as a part of this response, although it is not clear whether the ubiquitin-proteasome pathway plays an active role in terms of defense.

Enrichment of the GOslim category "Sequence-Specific DNA Binding Transcription Factor Activity" was observed earlier in C. japonica (cluster 3 and cluster 7) and later in 

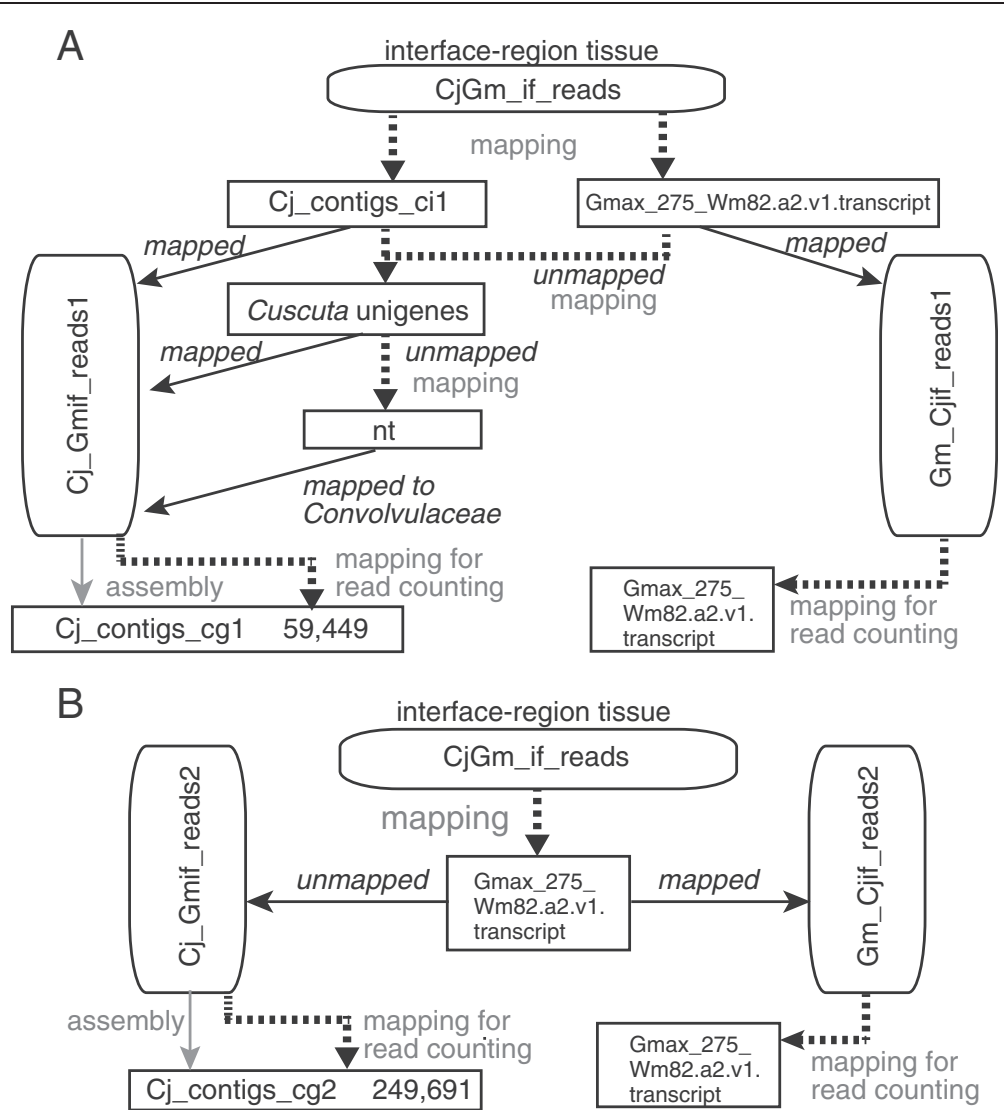

Figure 5 Schematic diagram of classification of reads from C. japonica-G. max interface region. Dotted lines indicate that mapping was performed. Solid lines indicate that assembly or classification was performed. Abbreviations: Cj, C. japonica; Gm, G. max; nc, not-in-contact; if, interface. (A) Stepwise classification. (B) Reference-based classification.

G. $\max$ (cluster 5), suggesting that $C$. japonica led the initiation of cellular changes in the interface region. Twentyseven C. japonica- and six G. max contigs were included in this category. Among them, Cj_contigs1.4_05837_00791 and Glyma.02G066200.1 exhibited similarity to the ERF BUD ENHANCER (EBE) gene, which positively regulates cell proliferation [27]. This observation tempted us to hypothesize that $E B E$ plays distinct roles in the parasite and host plants at different stages of parasitism.

Enrichment of the GOslim category "Photosynthesis" was an inevitable consequence of our experimental setting in which plants were transferred from continuous dark to the light/dark cycle after 48 haa. The GOslim categories of "Transporter Activity" and "Transport" were enriched in cluster 4 of $C$. japonica and G. max, respectively, and up-regulation of various transporter genes was observed. Increase in the transcriptional activity of transporter genes probably coincided with the increase in sink activity of C. japonica.

Modification of the cell wall is one of the key processes in establishing a cellular connection between parasite and host. Expansin belongs to the group of cell wall modifying proteins responsible for cell wall extension under acidic conditions. Tomato expansin gene, LeEXPA5, has been reported to be upregulated in root during syncytia formation by potato cyst nematode [28]. Cell wall disassembly by nematode triggers expression of host cell wall modifying proteins. To test whether a plant parasite also triggers expression of the host's cell wall modifying proteins, we investigated the temporal difference between the genes encoding the parasite's pectate lyase, a cell wall degrading enzyme, and the host's expansin, a cell wall modification protein. Expression levels of $5 \mathrm{C}$. japonica contigs that exhibited similarity to pectate lyase ( $e$ value $<1 \mathrm{e}-10$ ) peaked at 24 haa or 48 haa and then decreased (Figure 6C, left). We identified 23 G. max contigs that exhibited similarity to LeEXPA5 ( $e$ value $<1 \mathrm{e}-10)$. The expression levels of these contigs peaked at 72-, 96- or 120-haa (Figure 6C, right). These results demonstrated that the expression of cell wall degrading enzyme genes in $C$. japonica preceded the expression of expansins in G. max. Simultaneous profiling of gene expression in both parasite and host allowed us to monitor the temporal differences, and helped inferring the coordination or causal relationship between cellular processes in the two plants. 
Table 4 De novo assembly and annotation of $C$. japonica contigs classified in two different approaches

\begin{tabular}{|c|c|c|}
\hline & Cj_contigs_cg1 & Cj_contigs_cg2 \\
\hline Library type & $\begin{array}{l}\text { Illumina, } 74 \text { bp } \\
\text { single-end }\end{array}$ & $\begin{array}{l}\text { Illumina, } 74 \text { bp } \\
\text { single-end }\end{array}$ \\
\hline Assembler & Velvet/Oases & Velvet/Oases \\
\hline Assembled reads & $141,089,611$ & $205,957,611$ \\
\hline Number of contigs & 59,449 & 249,621 \\
\hline Median contig length & $325 \mathrm{bp}$ & $187 \mathrm{bp}$ \\
\hline Average contig length & $648 \mathrm{bp}$ & $354 \mathrm{bp}$ \\
\hline \multicolumn{3}{|l|}{ Length distribution (\%) } \\
\hline$<200 \mathrm{bp}$ & $18,009(30.3)$ & $134,968(54.1)$ \\
\hline 201-500 bp & 19,803 (33.3) & $75,199(30.1)$ \\
\hline 501-1000 bp & $9,739(16.4)$ & $22,305(8.9)$ \\
\hline$>1001$ bp & $11,898(20.0)$ & $17,149(6.9)$ \\
\hline \multicolumn{3}{|c|}{$\begin{array}{l}\text { Length distribution of contigs } \\
\text { no-hits-found in refseqplant (\%) }\end{array}$} \\
\hline$<200 \mathrm{bp}$ & $13,986(23.5)$ & $114,864(46.0)$ \\
\hline 201-500 bp & $12,232(20.6)$ & $53,096(21.3)$ \\
\hline 501-1000 bp & $3,160(5.3)$ & $10,616(4.3)$ \\
\hline$>1001$ bp & $952(1.6)$ & 2,639 (1.1) \\
\hline
\end{tabular}

\section{Conclusion}

We demonstrated that simultaneous analysis of gene expression profiles in a non-model parasitic plant, $C$. japonica, and a non-model host plant, I. balsamina, can be performed using RNA-Seq reads obtained from an interface region containing cells of both plants. We performed stepwise classification of reads using sequences of the plant species under study, plants belonging to the same genus, and, finally, plants in the same family. Using reads classified in this way, we de novo assembled the transcriptome sequence sets of the interface region. To confirm the annotation, we also assembled the transcriptome of $C$. reflexa. Applying a competitive mapping method, we could assess the quality of the performed classification. This assessment revealed that we achieved classification of reads with a misclassification rate low enough to be used reliably for analysis of differential expression of genes. We applied this read classification method to simultaneously analyze gene expression profiles in the non-model parasitic plant C. japonica and the model host plant G. max. We assembled the $C$. japonica transcriptome from reads classified by our stepwise classification approach. Using our $C$. japonica transcriptome in combination with the G. max reference transcriptome, we were able to robustly identify differentially expressed genes in both parasite and host. This simultaneous monitoring of gene expression in both parasitic and host plants shed new lights on coordination of cellular processes between two plants. This approach may be applicable to other multi-organism systems.

\section{Methods}

\section{Plant materials}

I. balsamina was grown on soil (Sukoyaka-Baido, Yanmar Co. Ltd., Osaka, Japan) mixed with the same volume of vermiculite (GS30L, Nittai, Osaka, Japan) in a $16 \mathrm{~h} / 8 \mathrm{~h}$ light/dark cycle at $25^{\circ} \mathrm{C}$. C. japonica seeds were dipped in concentrated sulfuric acid for 15 min, washed with distilled water and plated on wet glass filter paper (GA-100, Toyo Roshi Kaisha, Ltd., Tokyo, Japan) in the dark at $25^{\circ} \mathrm{C}$. Parasitism was induced by attaching the subapical region of C. japonica to the stem of I. balsamina and

Table 5 Assessment for the rate of misclassification of reads by mapping $C_{j} \_n c \_r e a d s$ and $G m \_n c \_r e a d s$ onto two contig sets

\begin{tabular}{|c|c|c|c|c|}
\hline \multirow[t]{7}{*}{ A. } & Contig set & \multicolumn{3}{|c|}{ Merged set of Cj_contigs_cg1 and Gmax_275_Wm82.a2.v1.transcript } \\
\hline & Classification & Reads mapped onto C. japonica (\%) & Reads mapped onto G.max (\%) & $A \cup C^{b}$ \\
\hline & Mapped reads (number) & \multicolumn{2}{|l|}{ Number of reads (\%)) } & \\
\hline & Cj_nc_reads & $54,936,999$ & 187 & 0.999 \\
\hline & $(114,056,994)$ & $(48.2)$ & $(0.00016)$ & \\
\hline & Gm_nc_reads & 1,791 & $25,880,696$ & \\
\hline & $(28,728,782)$ & $(0.0062)$ & $(80.9)$ & \\
\hline \multirow[t]{7}{*}{ B. } & Contig set & \multicolumn{2}{|c|}{ Merged set of Cj_contigs_cg2 and Gmax_275_Wm82.a2.v1.transcript } & \\
\hline & Classification & Reads mapped onto C. japonica (\%) & Reads mapped onto G.max (\%) & $A \cup C^{b}$ \\
\hline & Mapped reads (number) & \multicolumn{2}{|l|}{ Number of reads (\%) } & \\
\hline & Cj_nc_reads & $52,486,992$ & 191 & 0.955 \\
\hline & $(114,056,994)$ & $(46.0)$ & $(0.00017)$ & \\
\hline & Gm_nc_reads & $2,377,249$ & $24,253,863$ & \\
\hline & $(28,728,782)$ & $(8.27)$ & $(84.4)$ & \\
\hline
\end{tabular}

${ }^{a}$ Numbers indicate percentage of mapped reads to the total number of resds. ${ }^{b} A U C ;$ area under receiver operating characteristic (ROC) curve.

Mapping parameter was as follows; In Panel A and Panel B, match length $\geq 90$ bp and at most 1 mismatch and 1 gap allowed. 
Table 6 Assessment of the accuracy in identifying differentially expressed genes in interface regions of $C$. japonica-G. max association

\begin{tabular}{|c|c|c|c|c|}
\hline C.japonica read & \multicolumn{2}{|c|}{ Cj_Gmif_reads1 } & \multicolumn{2}{|c|}{ Cj_Gmif_reads2 } \\
\hline G. max read & \multicolumn{2}{|c|}{ Gm_Cjif_reads1 } & \multicolumn{2}{|c|}{ Gm_Cjif_reads2 } \\
\hline C. japonica contig & \multicolumn{2}{|c|}{ Cj_contigs_cg1 } & \multicolumn{2}{|c|}{ Cj_contigs_cg2 } \\
\hline G. max contig & \multicolumn{2}{|c|}{ Gmax_275_Wm82.a2.v1.transcript } & \multicolumn{2}{|c|}{ Gmax_275_Wm82.a2.v1.transcript } \\
\hline Mapping procedure & Separately ${ }^{\mathrm{a}}$ & Merged $^{\mathrm{b}}$ & Separately ${ }^{a}$ & Merged $^{\mathrm{b}}$ \\
\hline \multirow[t]{5}{*}{ Differentially expressed genes ${ }^{c}$ in C. japonica in G. max } & \multicolumn{2}{|c|}{ number of contigs (\%) ${ }^{d}$} & \multicolumn{2}{|c|}{ number of contigs $(\%)^{d}$} \\
\hline & 3,819 & 3,819 & 10,806 & 10,806 \\
\hline & $(6.4)$ & $(6.4)$ & $(4.3)$ & $(4.3)$ \\
\hline & 17,653 & 17,656 & 17,653 & 17,526 \\
\hline & (19.9) & $(19.9)$ & $(19.9)$ & $(19.7)$ \\
\hline
\end{tabular}

Indicated reads were mapped onto $C$. japonica contig and G. max contig separately, and uniquely hit reads to each contig set was used to estimate gene expression level. ${ }^{b}$ Indicated reads were mapped onto a merged contig set of $C$. japonica contig and $G$. max contig. If a given read hit to wrong contigs ( $C$. japonica read to G. max contig, or vice versa) that read was excluded from the estimation of gene expression level. 'Differentially expressed genes detected by using TCC

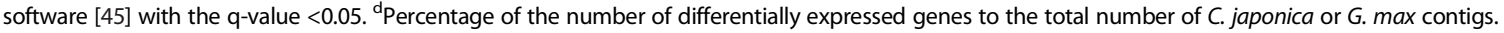

illuminating the junction with far red light (FL20S - FR-74, Toshiba, Tokyo, Japan) for $2 \mathrm{~h}$ [29]. After the far-red-light illumination, the plants were kept in darkness $48 \mathrm{~h}$ [30].

The interface region containing both $C$. japonica and I. balsamina tissues was harvested from the secondary C. japonica-I. balsamina association (Figure 1). A subapical region, 1-2 cm below the apical tip, of a 7-day-old C. japonica seedling grown in vermiculite (GS30L, Nittai, Osaka, Japan) in a $16 \mathrm{~h} / 8 \mathrm{~h}$ light/dark cycle at $25{ }^{\circ} \mathrm{C}$ was attached to the stem of the first 40-day-old I. balsamina, and parasitism was induced as described above. After 14 days, the subapical region extending from the first interface region was attached to the stem of the second 40-day-old I. balsamina, and parasitized as described above. The material from the secondary interface region containing both $C$. japonica and I. balsamina tissues was sampled after the $24 \mathrm{~h}$-dark treatment in the middle of

Table 7 Number of differentially expressed genes in each cluster

\begin{tabular}{llllll}
\hline Cluster & \multicolumn{3}{l}{$\begin{array}{l}\text { Number of differentially } \\
\text { expressed genes (\%) }\end{array}$} & Description \\
\cline { 2 - 5 } & \multicolumn{3}{c}{ C. japonica } & \multicolumn{3}{c}{ G. max } & \\
\hline 1 & 1,666 & $(43.6)$ & 4,940 & $(28.0)$ & max. at 24 haa \\
2 & 1,024 & $(26.8)$ & 8,828 & $(50.0)$ & max. at 72 haa \\
3 & 326 & $(8.5)$ & 489 & $(2.8)$ & max. at 48 haa \\
4 & 355 & $(9.3)$ & 2,866 & $(16.2)$ & max. at 120 haa \\
5 & 68 & $(1.8)$ & 67 & $(0.4)$ & max. at 96 haa \\
6 & 99 & $(2.6)$ & 82 & $(0.5)$ & max. at 72 haa, min. at 48 haa \\
7 & 184 & $(4.8)$ & 222 & $(1.2)$ & max. at 72 haa \\
8 & 63 & $(1.6)$ & 127 & $(0.7)$ & max. at 24 haa, min. at 72 haa \\
9 & 23 & $(0.6)$ & 18 & $(0.16)$ & max. at 72 haa, min. at 48 haa \\
10 & 11 & $(0.28)$ & 4 & $(0.02)$ & max. at 72 haa, min. at 96 haa \\
\hline
\end{tabular}

48 h-dark treatment [30]. In order to obtain not-in-contact (nc) C. japonica samples, the sub-apical region of an 8- to 10-day-old C. japonica was attached to a plastic rod (diameter $5 \mathrm{~mm}$ ) and subjected to the far-red light treatment and subsequent dark treatment. Subsequently, subapical region, 1-2 $\mathrm{cm}$ below the apical tip, was harvested. To obtain not-in-contact $I$. balsamina tissue, the stem of a 40-day-old I. balsamina was coiled two turns using a plastic-coated wire. Stem was harvested after the far-red light treatment and subsequent darkness treatment.

Soybean (Glycine max cv. Fukuyutaka) was sown on soil (Sukoyaka-Baido, Yanmar Co. Ltd.) mixed with the same volume of vermiculite (GS30L, Nittai) and grown in a $16 \mathrm{~h} / 8 \mathrm{~h}$ light/dark cycle at $25^{\circ} \mathrm{C}$. A 14-day-old G. max was parasitized by a 8 - to 10-day-old $C$. japonica at the stem part between cotyledon and the first foliage leaf. Parasitism was induced as described above (Figure 4A). The interface region containing both $C$. japonica and G. max tissues was harvested at five stages, 24 hours after attachment (haa), 48 haa, 72 haa, 96 haa and 120 haa. Three replicates were prepared for each stage.

Tomato plants (Solanum lycopersicum, cv. Moneymaker) serving as a host for C. reflexa were grown under greenhouse conditions (relative humidity $55 \%$, day temperature $25{ }^{\circ} \mathrm{C}$, night temperature $20{ }^{\circ} \mathrm{C}$, diurnal cycle: $16 \mathrm{~h}$ light $/ 8 \mathrm{~h}$ darkness, and light intensity $\left.190-600 \mu \mathrm{E} \cdot \mathrm{m}^{-2} \cdot \mathrm{s}^{-1}\right)$. The C. reflexa plants feeding on tomato stems were cut $(\sim 30 \mathrm{~cm}$ below the apex), transferred onto adult tomato stems, sprayed with water every 2 days, and covered with a plastic bag to facilitate the formation of haustorial connections. C. reflexa stems were harvested from $C$. reflexa individuals feeding on themselves or other individuals $>30 \mathrm{~cm}$ away from the nearest haustorial connection to a tomato host plant. All tissues were harvested with sterile razor blades, immediately frozen in liquid nitrogen, and stored at $-80{ }^{\circ} \mathrm{C}$. 


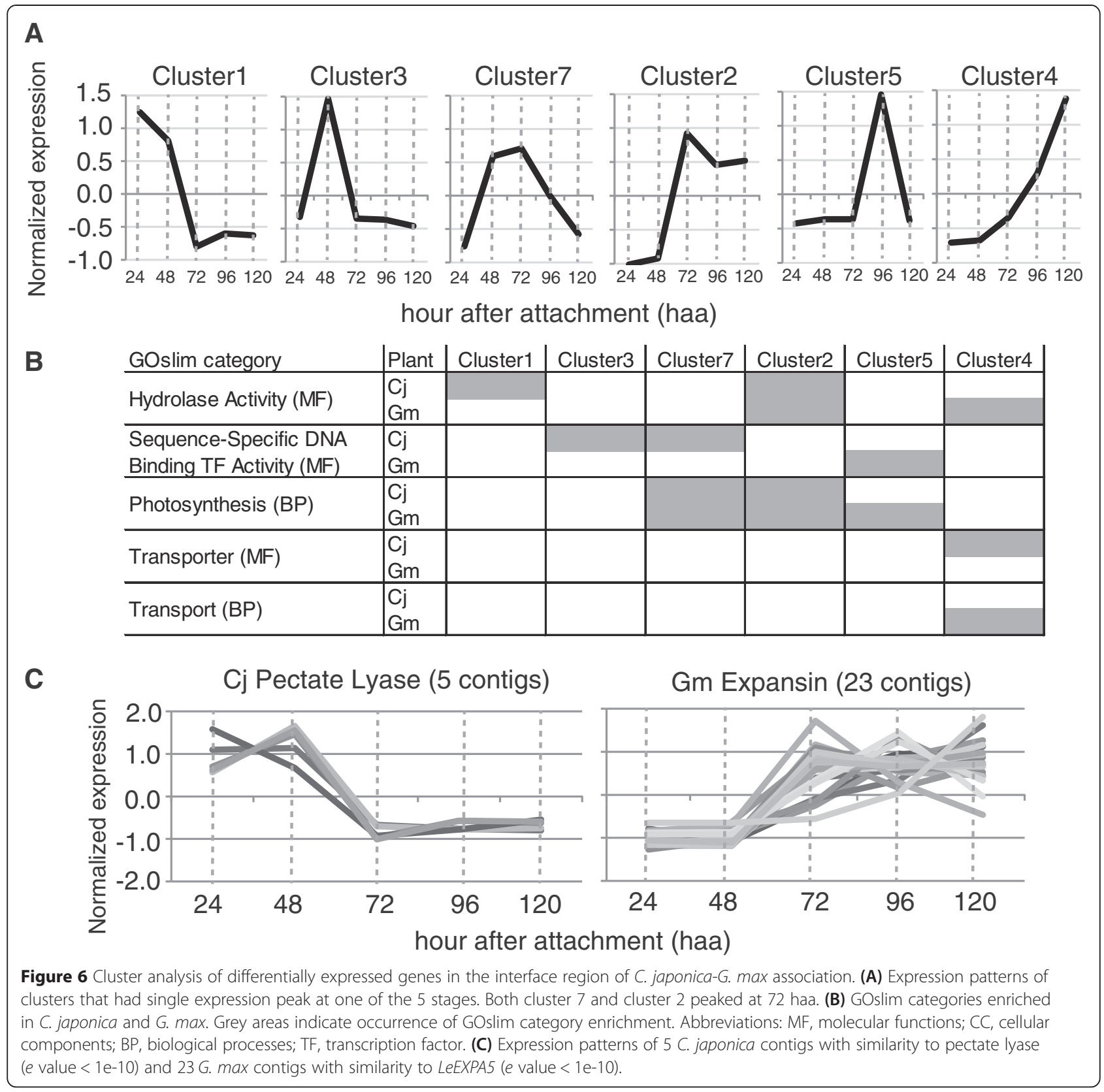

\section{RNA extraction, preparation of the sequencing library, and RNA-Seq}

Total RNA extraction was performed using the Qiagen RNeasy Plant Kit (cat. \# 74193, Qiagen, Netherlands) according to the manufacturer's protocol. RNA integrity was confirmed using the Agilent 2100 BioAnalyzer (Agilent Technologies, Santa Clara, CA, USA). In experiments using C. japonica and I. balsamina, RNA-Seq libraries were prepared using Illumina's TruSeq RNA Sample Prep Kit (RS-122-2001, RS-122-2002, Illumina Inc. San Diego, CA, USA) according to the manufacturer's standard protocol. Three libraries were sequenced in one full lane on the Illumina HiSeq 2000 platform, and 101-bp paired-end reads were obtained from Hokkaido System Science Co., Ltd (Sapporo, Japan). The data on C. japonica and I. balsamina reads were registered in DNA Data Bank of Japan (DDBJ) Read Archive (http://trace.ddbj.nig.ac.jp/dra/) [31] [DRA:DRR021687, DRR021688 and DRR021689 in DRA002408]. RNA-Seq library of not-in-contact tissue of G. $\max$ was prepared by the same procedure.

In experiments using C. japonica and G. max, RNASeq libraries were prepared using NEBNext $^{\circ}$ Ultra $^{\text {tu }}$ RNA Library Prep Kit for Illumina ${ }^{\circ}$ and NEBNext ${ }^{\circ}$ Poly(A) 
mRNA Magnetic Isolation Module (E7490S, E7530S, Illumina Inc.). Each library was sequenced on the Genome Analyzer II (Illumina Inc.) in one full lane to yield 74 bp single-end reads. The data on $C$. japonica and G. max reads were registered in DDBJ Read Archive (http://trace.ddbj.nig.ac.jp/dra/) [31] [DRA: DRR030860, DRR030861, DRR030862, DRR030863 and DRR030864].

Total RNA extraction from C. reflexa samples was performed by grinding cut plant material in liquid nitrogen with immediate addition of the TRIzol Reagent (Invitrogen, Carlsbad, CA, USA; $0.5 \mathrm{~mL}$ per $100 \mathrm{mg}$ tissue) as described previously [32]. After centrifugation $(10,000 \times \mathrm{g}$, $10 \mathrm{~min}$, at $4{ }^{\circ} \mathrm{C}$ ), the supernatant was transferred to a new tube, and an equal volume of phenol:chloroform:isoamyl alcohol (25:24:1, pH8.0; Roche, Basel, Switzerland) was added along with $1 \mu \mathrm{l}$ RNasin (Promega, Fitchburg, WI, USA). The mixture was centrifuged at $10,000 \times \mathrm{g}$ for $10 \mathrm{~min}$ at $4{ }^{\circ} \mathrm{C}$. The resulting supernatant was transferred to a new RNase-free plastic tube and extracted once with $200 \mu \mathrm{L}$ and once with $50 \mu \mathrm{L}$ of chloroform. To precipitate the total RNA, the supernatant was mixed with 2 volumes of $100 \%$ isopropanol, $1 / 10$ volume of $3 \mathrm{M}$ sodium acetate ( $\mathrm{pH}$ 5.2), and $1 \mu \mathrm{g}$ of linear acrylamide (Invitrogen), and the mixture was incubated for $>1 \mathrm{~h}$ at $-20{ }^{\circ} \mathrm{C}$. After centrifugation $\left(16,000 \times \mathrm{g}, 30 \mathrm{~min}\right.$, at $\left.4{ }^{\circ} \mathrm{C}\right)$, the resulting pellet was washed twice with $80 \%$ ethanol, once with $99 \%$ ethanol, air dried, and resuspended in $20 \mu \mathrm{L}$ of RNase-free water. To determine RNA quality and concentration, $1 \mu \mathrm{L}$ of the RNA samples was subjected to agarose gel electrophoreses ( $2 \%$ agarose, $1 \times$ Tris-borate-EDTA [TBE] buffer) and was quantified using a NanoDrop device (Thermo Fisher Scientific, Waltham, MA, USA). The libraries were sequenced on the Illumina HiSeq 2000 platform, and 90-bp paired-end reads were obtained from the Beijing Genomics Institute (BGI; Shenzhen, China). The data of C. reflexa reads were registered in the NCBI Sequence Read Archive (http://www.ncbi.nlm.nih.gov/Traces/sra) [33] [SRA: SRR1171084].

\section{Preprocessing of raw reads}

Read sets obtained from not-in-contact tissues of C. japonica, I. balsamina, and the interface region tissues were subjected to adapter removal, and to quality filtering using CASAVA ver.1.8.1 (Illumina) [34]. The read sets were filtered against a dataset of plant transfer RNA and ribosomal RNA sequences obtained from GenBank (gbpln[1-63].seq.gz, September 30, 2013) [35]. Reads that could be matched ( $e$ value $\leq 1 \mathrm{e}-5$ ) to this in-house dataset using BLASTN [36] were removed. Furthermore, read pairs spanning $<175 \mathrm{bp}$ were also removed. This procedure yielded 3 read sets $(\mathrm{Cj}$ _nc_reads, Ib_nc_reads and CjIb_if_reads; Figure 1).

Read sets obtained from the interface regions of $C$. japonica and G. max were subjected to adapter removal, and to quality filtering using CASAVA ver.1.8.2 (Illumina). The read sets were filtered against the transfer RNA and ribosomal RNA sequences as above. This procedure yielded read sets of the five stages (CjGm_if_reads; Figure $5 \mathrm{~A}$ and $5 \mathrm{~B})$.

Sequenced reads from samples of autofeeding C. reflexa growing on tomato samples were quality-trimmed and Illumina adapter sequences were removed using Trimmomatic [37] with default settings. PolyA-tails were removed from the ends of reads and read pairs with 1 or both reads $<75$ bp were discarded. The surviving reads were subjected to a filtering pipeline using bwa 0.75 [38] (bwa aln $-\mathrm{n} 1$ and otherwise default settings, followed by bwa sampe with default settings). In order to remove potential contamination by the tomato host plant, reads were aligned against ITAG2.3 cDNA [39]. The reads that survived this filter (i.e., neither read in a pair aligned properly to a filter sequence) were aligned against a database of common contaminants consisting of cDNA from $\mathrm{H}$. sapiens (GRCh37.75) as well as fungal and $E$. coli sequences obtained from Refseq (July 25, 2014).

\section{Assembly of contigs using reads from not-in-contact tissues} For assembly of transcriptome sets of the nc tissues of C. japonica (C)_nc_reads) and I. balsamina (Ib_nc_reads), these read sets were used as input for the Velvet software (version 1.2.10) [18] and, subsequently, Oases (version 0.2.08) [19]. The k-mer hash length of Velvet was set to 59 , and -ins length of Oases was set to 175 . The resulting transcript sets are referred to as Cj_nc_contigs and Ib_nc_contigs, respectively.

\section{Classification of the reads}

C. japonica and I. balsamina reads among the CjIb_if_ reads were classified using three sequential similarity searches (Figure 1). First, we performed de novo assembly of reads obtained from the nc samples as described above. CjIb_if_reads were mapped against the resulting transcript sets (Cj_nc_contigs and Ib_nc_contigs), using BLASTN (match length $\geq 90 \mathrm{bp}, e$ value $<1 \mathrm{e}-20$, allowing for $\leq 1$ mismatch and 1 gap). Reads that uniquely mapped to either $\mathrm{C}$ _nnc_contigs or Ib_nc_contigs were regarded as reads originating from $C$. japonica $\left(\mathrm{Cj}_{-} \mathrm{Ibif}\right.$ reads) or I. balsamina (Ib_Cjif_reads), respectively. Reads that could not be mapped during this first step were mapped against unigene sets of the same genus (corresponding to C. reflexa, C. pentagona [17], and C. suaveolens [18]) using BLASTN (match length $\geq 90 \mathrm{bp}, e$ value $<1 \mathrm{e}-20$, identity $\geq 90 \%$ ). Reads matching any of these transcripts were classified as Cj_Ibif_reads. Reads that could not be mapped during this second step were used in a BLASTN search against the nt database (May 1, 2013). If the top five hits of a read were the entries from the Convolvulaceae plant species, then the read was classified as Cj_Ibif_reads. 
Analogously, if the top five hits were Balsaminaceae entries, then the read was classified as Ib_Cjif_reads. For de novo transcriptome assembly of C. japonica and I. balsamina, we selected all read pairs where classification of the mates was identical.

C. japonica and G. max reads among the CjGm_if_reads were classified using two approaches (Figure 5A and B). In the first approach, CjGm_if_reads were mapped against the contig sets - Cj_contigs_ci1 and Gmax_275_ Wm82.a2.v1.transcript.fa.gz downloaded from the PhytozomeV10 (http://phytozome.jgi.doe.gov/pz/portal.html; match length $\geq 67 \mathrm{bp}, e$ value $<1 \mathrm{e}-20$, allowing for $\leq 1$ mismatch and 1 gap) [26]. Reads that uniquely mapped to Gmax_ 275_Wm82.a2.v1.transcript were regarded as reads originating from G. max (Gm_Cjif_reads1). Reads that uniquely mapped to $\mathrm{Cj}$ _contigs_ci1 were regarded as reads originating from C. japonica (Cj_Gmif_reads1). Reads that could not be mapped during this first step were mapped against unigenes of Cuscuta genus (match length $\geq 67 \mathrm{bp}$, $e$ value $<1 \mathrm{e}-20$, identity $\geq 90 \%$ ). Reads mapped to Cuscutagenus unigenes were regarded as those originating from C. japonica and added to $\mathrm{Cj}_{\text {_Gmif_reads1. Reads that }}$ could not be mapped during this second step were used in a BLASTN search against the nt database (May 1, 2013). If the top 5 hits of a read were the entries from the Convolvulaceae plant species, then the read was classified as Cj_Gmif_reads1.

In the second approach, reads derived from G. max in CjGm_if_reads were separated by mapping CjGm_if_ reads onto Gmax_275_Wm82.a2.v1.transcript. Mapped reads were regarded as those derived from G. $\max$ (Gm_Cjif_reads2). Unmapped reads were regarded as those derived from $C$. japonica. The resulting $\mathrm{Cj}_{\text {_Gmif }}$ reads2 was used for assembly to obtain $\mathrm{Cj}_{-}$contigs_cg2.

\section{De novo transcriptome assembly}

Assembly of $C$. japonica contigs using a merged dataset of $\mathrm{Cj} \_n c \_r e a d s$ and $\mathrm{Cj}$ _Ibif_reads, and I. balsamina contigs using a merged dataset of Ib_nc_reads and Ib_Cjif_ reads, were performed by using the Velvet software (version 1.2.10) [19] and, subsequently, Oases (version 0.2.08) [20]. The k-mer hash length of Velvet was set to 59 , and -ins length of Oases was set to 175 . The resulting transcript sets are referred to as $\mathrm{Cj}_{\text {_contigs_ci1 or }}$ Ib_contigs, respectively. The read sets used for de novo assembly are available [DDBJ DRA: DRZ003178 and DRZ003179]. Sequence files of Cj_contigs_ci1 and Ib_contigs are available as Additional file 6 and Additional file 7.

In the de novo transcriptome assembly using $\mathrm{Cj}_{\text {_Gmif }}$ reads1 and $\mathrm{C}$ __Gmif_reads2, the same assembly procedure using Velvet/Oases as described above was used. The resulting transcript sets are referred to as $\mathrm{Cj}$ _contigs_cg1 and $\mathrm{Cj}_{-}$contigs_cg2, respectively. Sequence file of $\mathrm{Cj}_{-}$ contigs_cg1 is available as Additional file 8.
In the de novo transcriptome assembly of $C$. reflexa, all read pairs from $C$. reflexa that survived the filtering pipeline described above were used for de novo transcriptome assembly using Trinity (version r20140717 with default parameters and -jaccard_clip option) [21].

\section{Functional annotation}

Cj_contigs_ci1, Cj_contigs_cg1 and $\mathrm{Cj}$ _contigs_cg2 were searched against the plant protein database of refseqplant (e value <1e-5) using BLASTX [40]. GO annotations [41] were obtained from TAIR10 according to the similarity to Arabidopsis thaliana genes (ftp://ftp.arabidopsis.org/home/ tair/Genes/TAIR10_genome_release/). The contig sets were further matched against unigenes of $C$. pentagona [17], C. suaveolens [18], C. reflexa (SRA: SRP038020), Tryphysaria versicolor (TrVeBC1 and TrVeBC2), Striga hermonthica (StHeBC1 and StHeBC2), and Orobanche aegyptiaca (OrAeBC4) (Parasitic Plant Genome Project http://ppgp.huck.psu.edu/) using BLASTN with $e$ value $<1 \mathrm{e}-5$. Ib_contigs were used for BLASTX search against the plant protein database of refseqplant ( $e$ value $<1 \mathrm{e}-5$ ). According to the similarity to Arabidopsis genes, a GO annotation was obtained as described in Mochizuki et al. [40] using the GO dataset available at http://www.plant.osakafuu.ac.jp/ ogata/downloadgo.html. Prediction of ORFs was performed with the OrfPredictor software [42]. Fulllength transcripts were identified by testing whether both start and stop codon were detected within a contig's sequence. The assembled $C$. reflexa contigs were checked for ORFs using an in-house Python script (https://github. com/cschu/fortuna). Contigs with an ORF of at least 200 bp were then searched against the plant protein database of refseqplant using BLASTX. GO annotation was then attempted for all contigs that matched against refseqplant using the same data sets described above. C. reflexa BLASTX runs were performed with the following parameters: $e$ value $<1 \mathrm{e}-5, \geq 75 \%$ query coverage, $>40 \%$ identity (identities + positives).

\section{Gene expression analysis and identification of differentially expressed genes}

$\mathrm{Cj}$ _nc_reads and $\mathrm{Cj}$ _Ibif_reads were mapped to $\mathrm{Cj}$ _contigs_cil, and Ib_nc_reads and Ib_Cjif_reads were mapped to Ib_contigs using BLASTN (parameter settings: match length $\geq 90 \mathrm{bp}$; $\leq 1$ mismatch and 1 gap insertion allowed). Reads per kilobase per million mapped reads (RPKM) were calculated separately. Library size normalization and differential gene expression analysis were performed using the DESeq [43] and R software [44]. Cj_Gmif_reads1 and Cj_Gmif_reads2 were mapped to Cj_contigs_cg1 and Cj_contigs_cg2, respectively, using BLASTN (parameter settings: match length $\geq 90 \mathrm{bp} ; \leq 1$ mismatch and 1 gap insertion allowed). Gm_Cjif_reads1 and Gm_Cjif_reads2 were mapped to Gmax_275_Wm82.a2.v1.transcript using 
BLASTN (parameter settings: same as above). RPKM values were calculated separately.

\section{Assessment of read classification quality}

To evaluate the degree of misclassification of reads with respect to their source organism, Cj_nc_reads and Ib_nc_reads were mapped to a merged transcript set consisting of Cj_contigs_cil and Ib_contigs using BLASTN (match length $\geq 90 \mathrm{bp} ; e$ value $<1 \mathrm{e}-20,1$ mismatch and 1 gap insertion allowed). Cj_nc_reads that were mapped to Ib_contigs, and Ib_nc_reads that were mapped to $\mathrm{Cj}$ _contigs_cil were regarded as misclassified. To evaluate the rate of assignment of $C$. japonica reads to I. balsamina, or vice versa, Cj_nc_reads and Ib_nc_reads, respectively, were mapped to a merged transcript set consisting of $\mathrm{Cj}_{\text {_nc_contigs }}$ and $\mathrm{Ib}$ _nc_contigs using BLASTN as described above. Cj_nc_reads that were mapped to Ib_nc_contigs as well as Ib_nc_reads mapped to $\mathrm{Cj}_{-}$nc_contigs were regarded as misclassified. For the binary classification of $C$. japonica reads and $I$. balsamina reads, the following 4 outcomes are possible. We defined a $C$. japonica read as a true positive (TP) if it was mapped to a $C$. japonica contig, and as a false negative (FN) if it was mapped to an I. balsamina contig. An I. balsamina read that was mapped to a $C$. japonica contig was defined as a false positive (FP). Finally, an I. balsamina read that was mapped to an I. balsamina contig was defined as a true negative (TN). For the nomenclature of I. balsamina reads, switch the term " $C$. japonica" and "I. balsamina" in the definition above. The true positive rate (TPR) was defined as TP / (TP + FN) and false positive rate (FPR) as FP / (TN + FP). The ROC AUC was calculated using the R package ROCR [45]. The same procedure was applied to evaluate the degree of misclassification between C. japonica and G. max.

\section{Cluster analysis}

Read counts were normalized and subjected to identification of differentially expressed genes by using TCC and $\mathrm{R}$ software with FDR $<0.05$ [46]. Clustering analysis of differentially expressed genes was performed by using function hclust from the R stats package [47].

\section{Light microscopy}

The interface tissues were fixed with formalin, acetic acid:ethanol:water (90:5:5, v/v/v). Fixed samples were sliced into 80 - 100 micrometer-thick sections with the Vibratome (VIB-1500, Vibratome Co. Ltd., St. Louis, MO, USA). Histochemical staining of sections was performed using a $0.5 \%(\mathrm{w} / \mathrm{v})$ solution of Toluindine Blue O (1B-481, Waldeck $\mathrm{GmbH} \&$ Co., Munster, Germany) in distilled water. Stained slices were observed and photographs were taken by using the Biological Microscope BX51 (Olympus, Tokyo, Japan) with the CCD camera, VB-7010 (KEYENCE, Osaka, Japan).

\section{Additional files}

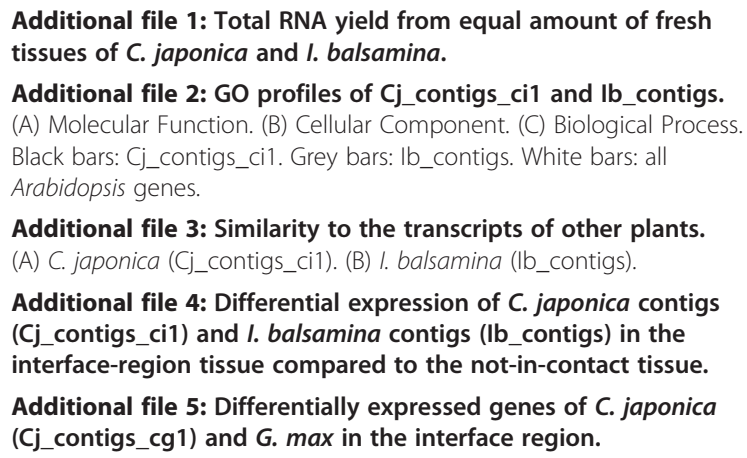

Additional file 2: $\mathrm{GO}$ profiles of $\mathrm{Cj}$ _contigs_ci1 and Ib_contigs. (A) Molecular Function. (B) Cellular Component. (C) Biological Process. Black bars: Cj_contigs_ci1. Grey bars: Ib_contigs. White bars: all Arabidopsis genes.

Additional file 3: Similarity to the transcripts of other plants. (A) C. japonica (Cj_contigs_ci1). (B) I. balsamina (Ib_contigs).

Additional file 4: Differential expression of $C$. japonica contigs (Cj_contigs_ci 1 ) and I. balsamina contigs (Ib_contigs) in the interface-region tissue compared to the not-in-contact tissue.

Additional file 5: Differentially expressed genes of $C$. japonica (Cj_contigs_cg1) and G. max in the interface region.

Additional file 6: A multi FASTA file of the Cj_contigs_ci1 (140519_Cj_ contigs_ci1.fa).

Additional file 7: A multi FASTA file of the lb_contigs (140519_Ib_contigs.fa).

Additional file 8: A multi FASTA file of the $\mathrm{Cj}_{\text {_contigs_cg1. }}$

\section{Abbreviations}

Cj: Cuscuta japonica; Cr: Cuscuta reflexa; Ib: Impatiens balsamina; Gm: Glycine max; nc: not-in-contact; if: interface region; RPKM: Reads per kilobase per million mapped reads; ROC AUC: Receiver operating characteristic and its area under the curve.

\section{Competing interests}

The authors declare that they have no competing interests.

\section{Authors' contributions}

DI performed experiments, bioinformatics analysis (C. japonica, I. balsamina and G. max), light microscopy and participated in manuscript preparation. CS performed bioinformatics analysis and assembly of $C$. reflexa RNA-Seq reads and participated in manuscript preparation. WZ performed experiments with C. reflexa. YO performed assembly of RNA-Seq reads of $C$. japonica. TS and TK performed sequencing and assembly of $C$. japonica-G. max interface regions. TF performed experiments with C. japonica-G. max interface regions. FK conceived and designed experiment with C. reflexa, and participated in manuscript preparation. KA conceived this study, designed experiments and analyses, and participated in manuscript preparation. All authors read and approved the final manuscript.

\section{Acknowledgements}

We thank Kyoji Yamada and Tatsuya Wakasugi (Toyama University) for their generous gift of C. japonica seeds. We also thank Atsushi Okazawa (Osaka Prefecture University) for helpful discussion. This work was partly supported by the Grant-in-Aid for Scientific Research (A; No. 23248005) to KA, MPI-MPP internal funds to FK, and Grant-in-Aid for Scientific Research for Plant Graduate Student from the Nara Institute of Science and Technology supported by MEXT, Japan to DI.

\section{Author details}

${ }^{1}$ Graduate School of Life and Environmental Sciences, Osaka Prefecture University, 1-1 Gakuen-Cho, Naka-Ku, Sakai, Osaka 599-8531, Japan. ${ }^{2}$ Max Planck Institute of Molecular Plant Physiology, Wissenschaftspark Potsdam-Golm, Am Mühlenberg 1, Potsdam 14476, Germany. ${ }^{3}$ Plant Global Education Project, Graduate School of Biological Sciences, Nara Institute of Science and Technology (NAIST), 8916-5 Takayama, Ikoma 630-0192, Japan. ${ }^{4}$ Metabolic Systems Research Team, RIKEN Center for Sustainable Resource Science (CSRC), 1-7-22 Suehiro, Tsurumi, Yokohama 230-0045, Japan. ${ }^{5}$ Present Address: Bioinformatics Group, The Sainsbury Laboratory, Norwich Research Park, Norwich NR4 7UH, UK. ${ }^{6}$ Present Address: Department of Molecular Systems Biology (Ecogenomics and Systems Biology), Vienna University, Althanstraße 14, Vienna A-1090, Austria.

Received: 8 October 2014 Accepted: 12 March 2015

Published online: 03 May 2015 


\section{References}

1. Westwood JH, Yoder Jl, Timko MP, de Pamphilis CW. The evolution of parasitism in plants. Trends Plant Sci. 2010;15:227-35.

2. Barkman TJ, McNeal JR, Lim SH, Coat G, Croom HB, Young ND, et al. Mitochondrial DNA suggests at least 11 origins of parasitism in angiosperms and reveals genomic chimerism in parasitic plants. BMC Evol Biol. 2007;7:248.

3. Yoder JI, Scholes JD. Host plant resistance to parasitic weeds; recent progress and bottlenecks. Curr Opin Plant Biol. 2010;13:478-84.

4. McNeal JR, Arumugunathan K, Kuehl JV, Boore JL, Depamphilis CW. Systematics and plastid genome evolution of the cryptically photosynthetic parasitic plant genus Cuscuta (Convolvulaceae). BMC Biol. 2007;5:55.

5. Lee KB. Structure and development of the upper haustorium in the parasitic flowering plant Cuscuta japonica (Convolvulaceae). Am J Bot. 2007;94:737-45.

6. Nagar R, Singh M, Sanwal GG. Cell-Wall Degrading Enzymes in Cuscuta-Reflexa and Its Hosts. J Exp Bot. 1984:35:1104-12.

7. Runyon JB, Mescher MC, De Moraes CM. Plant defenses against parasitic plants show similarities to those induced by herbivores and pathogens. Plant Signal Behav. 2010;5:929-31.

8. Christensen NM, Dorr I, Hansen M, van der Kooij TA, Schulz A. Development of Cuscuta species on a partially incompatible host: induction of xylem transfer cells. Protoplasma. 2003;220:131-42.

9. Kubo M, Ueda H, Park P, Kawaguchi M, Sugimoto Y. Reactions of Lotus japonicus ecotypes and mutants to root parasitic plants. J Plant Physiol. 2009:166:353-62

10. Haupt S, Oparka KJ, Sauer N, Neumann S. Macromolecular trafficking between Nicotiana tabacum and the holoparasite Cuscuta reflexa. J Exp Bot. 2001;52:173-7.

11. Roney JK, Khatibi PA, Westwood JH. Cross-species translocation of mRNA from host plants into the parasitic plant dodder. Plant Physiol. 2007;143:1037-43.

12. Kim G, LeBlanc ML, Wafula EK, de Pamphilis CW, Westwood JH. Plant science. Genomic-scale exchange of mRNA between a parasitic plant and its hosts. Science. 2014;345:808-11.

13. Alakonya A, Kumar R, Koenig D, Kimura S, Townsley B, Runo S, et al. Interspecific RNA interference of SHOOT MERISTEMLESS-like disrupts Cuscuta pentagona plant parasitism. Plant Cell. 2012;24:3153-66.

14. Birschwilks M, Haupt S, Hofius D, Neumann S. Transfer of phloem-mobile substances from the host plants to the holoparasite Cuscuta sp. J Exp Bot. 2006;57:911-21.

15. Honaas LA, Wafula EK, Yang Z, Der JP, Wickett NJ, Altman NS, et al. Functional genomics of a generalist parasitic plant: laser microdissection of host-parasite interface reveals host-specific patterns of parasite gene expression. BMC Plant Biol. 2013;13:9.

16. Vaughn KC. Dodder hyphae invade the host: a structural and immunocytochemical characterization. Protoplasma. 2003;220:189-200.

17. Ranjan A, Ichihashi Y, Farhi M, Zumstein K, Townsley B, David-Schwartz R et al. De novo assembly and characterization of the transcriptome of the parasitic weed Cuscuta pentagona identifies genes associated with plant parasitism. Plant Physiol. 2014;166:1186-99.

18. Jiang $L$, Wijeratnen AJ, Wijeratne S, Frage M, Meulia T, Doohan D, et al. Profiling mRNAs of Two Cuscuta Species Reveals Possible Candidate Transcripts Shared by Parasitic Plants. PLoS One. 2013;8:e.81389.

19. Zerbino DR, Birney E. Velvet: algorithms for de novo short read assembly using de Bruijn graphs. Genome Res. 2008;18:821-9.

20. Schulz MH, Zerbino DR, Vingron M, Birney E. Oases: robust de novo RNA-seq assembly across the dynamic range of expression levels. Bioinformatics. 2012;28:1086-92

21. Grabherr MG, Haas BJ, Yassour M, Levin JZ, Thompson DA, Amit I, et al. Full-length transcriptome assembly from RNA-Seq data without a reference genome. Nat Biotechnol. 2011;29:644-52.

22. Pruitt KD, Tatusova T, Maglott DR. NCBI reference sequences (RefSeq): a curated non-redundant sequence database of genomes, transcripts and proteins. Nucleic Acids Res. 2007;35:D61-65.

23. Lamesch P, Berardini TZ, Li D, Swarbreck D, Wilks C, Sasidharan R, et al. The Arabidopsis Information Resource (TAIR): improved gene annotation and new tools. Nucleic Acids Res. 2012;40:D1202-1210.

24. Fawcett T. ROC graphs: notes and practical considerations for data mining researchers. In: Technical Report HPL-2003-4. Palo Alto, CA: HP Labs; 2003.

25. Schmutz J, Cannon SB, Schlueter J, Ma J, Mitros T, Nelson W, et al. Genome sequence of the palaeopolyploid soybean. Nature. 2010;463:178-83.
26. Goodstein DM, Shu S, Howson R, Neupane R, Hayes RD, Fazo J, et al. Phytozome: a comparative platform for green plant genomics. Nucleic Acids Res. 2012;40:D1178-1186.

27. Mehrnia M, Balazadeh S, Zanor MI, Mueller-Roeber B. EBE, an AP2/ERF transcription factor highly expressed in proliferating cells, affects shoot architecture in Arabidopsis. Plant Physiology. 2013;162:842-57.

28. Fudali S, Sobczak M, Janakowski S, Griesser M, Grundler FM, Golinowski W. Expansins are among plant cell wall modifying agents specifically expressed during development of nematode-induced syncytia. Plant Signal Behav. 2008;3:969-71.

29. Tada Y, Wakasugi T, Nishikawa A, Furuhashi K, Yamada K. Developmental regulation of a gene coding for a low-molecular-weight heat shock protein during haustorium formation in the seedlings of a holoparasitic plant, Cuscuta japonica. Plant Cell Physiol. 2000;41:1373-80.

30. Tada Y, Sugai M, Furuhashi K. Haustoria of Cuscuta japonica, a Holoparasitic Flowering Plant, Are Induced by the Cooperative Effects of Far-Red Light and Tactile Stimuli. Plant Cell Physiol. 1996;37:1049-53.

31. Kodama Y, Shumway M, Leinonen R. The Sequence Read Archive: explosive growth of sequencing data. Nucleic Acids Res. 2012;40:D54-56.

32. Zhang S, Sun L, Kragler F. The phloem-delivered RNA pool contains small noncoding RNAs and interferes with translation. Plant Physiology. 2009;150:378-87.

33. Wheeler DL, Barrett T, Benson DA, Bryant SH, Canese K, Chetvernin V, et al. Database resources of the National Center for Biotechnology Information. Nucleic Acids Res. 2007;35:D5-12.

34. Hosseini P, Tremblay A, Matthews BF, Alkharouf NW. An efficient annotation and gene-expression derivation tool for Illumina Solexa datasets. BMC Res Notes. 2010;3:183.

35. Benson DA, Karsch-Mizrachi I, Clark K, Lipman DJ, Ostell J, Sayers EW. GenBank. Nucleic Acids Res. 2012:40:D48-53.

36. Altschul SF, Gish W, Miller W, Myers EW, Lipman DJ. Basic local alignment search tool. J Mol Biol. 1990;215:403-10.

37. Lohse M, Bolger AM, Nagel A, Fernie AR, Lunn JE, Stitt M, et al. RobiNA: a user-friendly, integrated software solution for RNA-Seq-based transcriptomics. Nucleic Acids Res. 2012;40:W622-627.

38. Li H, Durbin R. Fast and accurate short read alignment with Burrows-Wheeler transform. Bioinformatics. 2009:25:1754-60.

39. TheTomatoGenomeConsortium. The tomato genome sequence provides insights into fleshy fruit evolution. Nature. 2012;485:635-41.

40. Mochizuki T, Ogata Y, Hirata Y, Ohki ST. Quantitative transcriptional changes associated with chlorosis severity in mosaic leaves of tobacco plants infected with Cucumber mosaic virus. Mol Plant Pathol. 2014;15:242-54.

41. Ashburner M, Ball CA, Blake JA, Botstein D, Butler H, Cherry JM, et al. Gene ontology: tool for the unification of biology. The Gene Ontology Consortium. Nat Genet. 2000;25:25-9.

42. Min XJ, Butler G, Storms R, Tsang A. OrfPredictor: predicting protein-coding regions in EST-derived sequences. Nucleic Acids Res. 2005;33:W677-680.

43. Anders $\mathrm{S}$, Huber W. Differential expression analysis for sequence count data. Genome Biol. 2010;11:R106.

44. Ihaka R, Gentleman R. R: A language for data analysis and graphics. J Comput Graph Stat. 1996:5:299-314

45. Sing T, Sander O, Beerenwinkel N, Lengauer T. ROCR: visualizing classifier performance in R. Bioinformatics. 2005;21:3940-1.

46. Sun J, Nishiyama T, Shimizu K, Kadota K. TCC: an R package for comparing tag count data with robust normalization strategies. BMC Bioinformatics. 2013;14:219

47. Paradis E, Claude J, Strimmer K. APE: Analyses of Phylogenetics and Evolution in R language. Bioinformatics. 2004;20:289-90. 